\title{
The New Wireless LinguControl
}

\author{
Walter Schmitt, Werner Zang \\ Klinikum RWTH Aachen, Fachhochschule Aachen, Germany
}

\begin{abstract}
Persons who do not have the use of their limbs are isolated from daily functioning in society. It has been found that pathologies like traumatic injuries of the spinal cord or diseases of the neuromuscular and central nervous system do not affect the function of the user's tongue. LinguControl is a tongue activated communication controller and was presented at the $3 \mathrm{rd}$ ICCH. The intraorally worn thin plate with encapsulated sensors was connected by cable with an extra-oral smart box having computing means. The New Wireless LinguControl version is an intraoral assembly with telemetric based transmitting of the tongue position to the microcomputer operated controller. The embodiment of the controller includes software for decoding the received signals and for converting them into infrared control signals to manipulate environmental devices.
\end{abstract}

\section{Introduction}

The number of disabled people is increasing in the general population. Some of these persons may have suffered traumatic injuries of their spinal cord, such as during automobile accidents or sport injuries, progressive neuromuscular diseases, such as multipe sclerosis and the like.

Rehabilitation technology offers a great amount of communication devices:

Mouthstick controller, a device clenched in the users teeth, leads easily to detorisation of teeth and oral occlusion. Additionally, a mouthstick controller requires a high degree of mobility to accomplish specific tasks.

Voice recognition systems require good articulation and volume. In many cases the speech articulation and volume of quadriplegics is hampered.

Single switch controlled devices require an action of breathin, chin movement and this like. A single switch controller does not achieve the desired speed to handle modern communication systems.

To have a near-normal lifestyle, there is a need for faster and more aesthetically acceptable communications controller. The New LinguControl is a universal intraoral lingually activated communication controller, that can be used by a large number of persons having limited mobility, to operate various devices. 


\section{Method}

Eleven sensors are integrated in a $2 \mathrm{~mm}$ thin smooth plate, which covers the hard palate and a part of the vestibule of the upper jaw. The basis for the plate is Bioplast, a biocompatible material used in dentistry. Two kind of sensors, an optical and a mechanical sensor were developped to fufil the selection criteria for intraoral applications. Furthermore The New LinguControl (Fig.1) includes intraoral electronics for encoding the sensor signals and a radio-based transmitting assembly (Fig. 2).

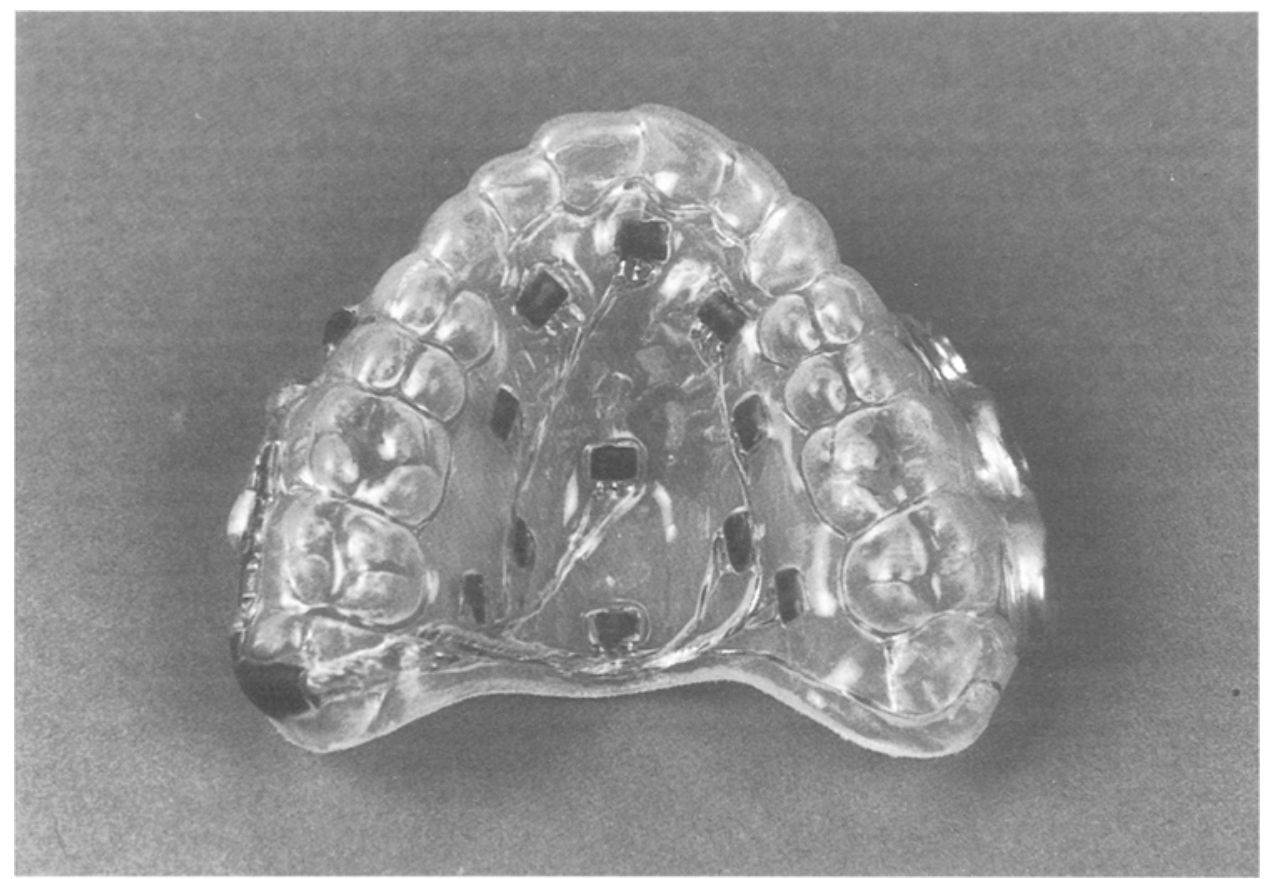

Fig. 1 The New Wireless LinguControl plate

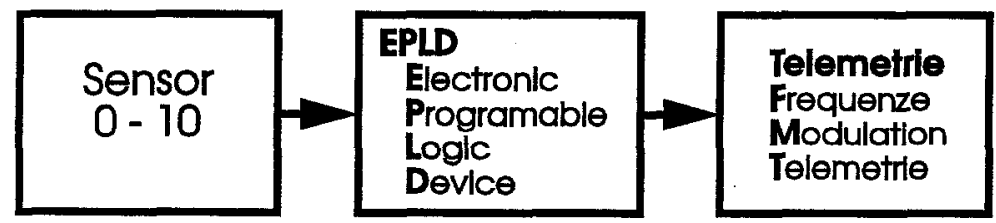

Fig. 2 Diagram of intraoral electronic device 
The extraoral controller includes a receiver for decoding the encoded signals.

A Mikrocomputer then forms a set of instructions and sends commands to the operating devices by infrared remote control (Fig.3). While using the cable connected LinguControl version, the user had to touch one of the eleven sensors with his tongue to activate a control command. Comparing it to the new radio-based version, while using the optical sensors, there is an important additional function. Here the tongue don't has to touch the sensors any more. By meassuring the reflected radiation of all eleven light barrier sensors, the telemetry transmits the intraoral positon of the tongue to the microcomputer operated controler. That means, that every position of the tongue in the mouth can be controlled by the eleven sensors. The next step is to programm tongue positions and to transform them into commands. This is done by the software LinguLearn.
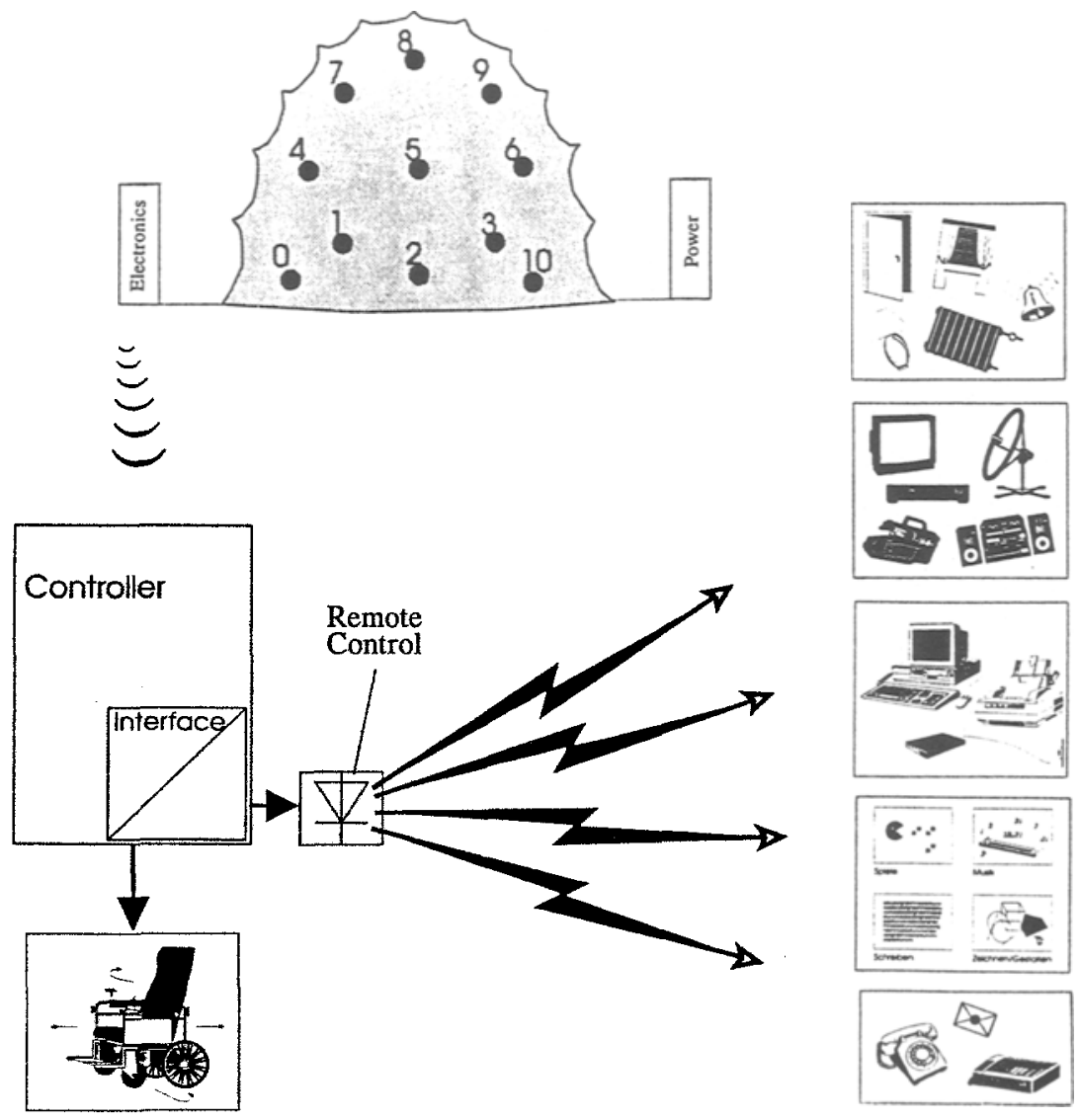

Fig. 3 Diagram of the New Wireless LinguControl system 
For programming the system, the user has to touch a reproducable position in the mouth. The intensity of the radiation of the eleven sensors marks this position and the data is fixed in the controller. Howmany commands can be programmed depend of the mobilility of the users tongue and the users demands (Fig.4).

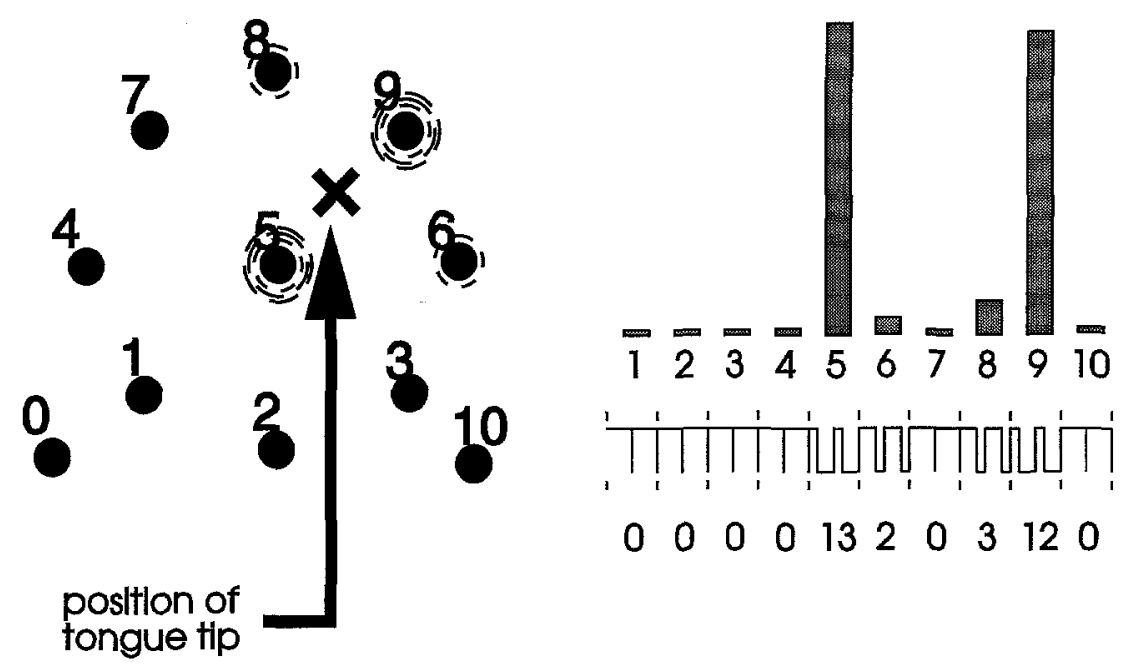

Fig. 4 Reflected light intensity and code of transmitted data for position of tongue tip

\section{Results}

While The New Wireless LinguControl System needs more intraoral electronics, a clinical study was realized to find out, where to place the electronic components. The study has shown, that there is enough space in the vestibule of the maxilla.

The dental plate is quickly accepted by the user in the same manner as other dental prothesis. To run the LinguControl system, the microcomputer operated smart box can be fixed at the wheelchair and powered by the wheelchair energy system. That makes the user independent from any outside cable connection. Error-free activation of the functions is learnt in the shortest of time. 
While sitting in the wheelchair, the remote control intercom-phone and the PC-FaxModem make modern information exchange accessible. LinguControl can instruct the telephone to send and receive calls; phone numbers may be stored and redialed automatically.

Television sets, videorecorders, videocameras, satellite dishes or hi-fi receivers, compact disc players and many more electronic entertainment devices can be switched by the universal IR remote control.

Basic and special functions of the particular device can be programmed into LinguContol, depending on the users individual desires. LinguControl replaces the computer keyboard as well as the computer mouse with wireless remote control. The system is independent of special interface software. It works with simple text editors as well as with complex programms. It opens up more fascinating and creative applications of computers. Music can be composed, changed and repayed in full orchestral sound. Colored graphics cam be painted. Last, but not least, skill or adventure games may be played alone or in company.

\section{Discussion}

The LinguControl system is aimed to return the user to independence in as many social and vocational areas as possible.

For this to be achieved any of the descriebed applications can be implemented. Wheather driving an electric wheelchair, working on a computer or controlling domestic environment - LinguControl offers a multitude of possibilities.

The initial clinic results with regard to application and handling of the tongue controlled manipulator are more than satisfactory. The microcomputer controlled intraoral sensorfield provides an ideal means for the social and occupational rehabilitation of handicapped persons via application-oriented programs.

Ultimately, the range of the tongue controlled manipulator convers not only paraand tetraplegics, but all those, whose extremities fail to fulfil their biomedical functions. 